\title{
Oxygen Transfer and Power Consumption in an Aeration System Using Mist and Circulation Flow Generated by a Rotating Cone
}

\author{
Takahiro ADACHI* \\ Department of Mechanical Engineering, Akita University, Tegata-Gakuen 1-1, Akita 010-8502, Japan \\ TEL: +81-18-889-2306, FAX:+81-18-837-0405, E-mail: adachi@ipc.akita-u.ac.jp
}

\begin{abstract}
We have experimentally found the liquid film flow characteristics rising along the outer surface of a rotating cone. When the film flow goes up fully upward, the liquid film can not keep the filmwise condition and is eventually atomized into a mist flow. In this research, we apply the mechanism to atomize the liquid and to transport oxygen from the air to the water through the atomized water droplets. It is recognized that the mixing is effective in oxygen mass transfer. It is found that a dual effect of the mixing and mist flow by using a rotating cone increases the oxygen mass transfer because the mist flow has a small diameter and large surface area between liquid and gas. In addition, the flow pattern is changed from steady flow to unsteady one if the rotation rate increases further, where the oxygen mass transfer is slightly enhanced. Furthermore, a correlation between the unit volume power consumption and the oxygen mass transfer is examined and shows that the oxygen transfer increases in power law relationship with the unit volume power consumption in the case with the mist flow.
\end{abstract}

Keywords: Rotating Cone; Film Flow; Mist Flow; Dissolved Oxygen; Oxygen Mass Transfer; 


\section{Nomenclature}

Do : dissolved oxygen concentration (mg/L)

$k_{L} a$ : oxygen mass transfer rate $\left(\mathrm{h}^{-1}\right)$

$h_{1}$ : hight of the water surface from the bottom (mm)

$h_{2}$ : hight of the circular tank from the bottom (mm)

$P$ : power, $2 \pi \omega T / 60(\mathrm{~W})$

$r_{0}:$ radius of the circular plate $(\mathrm{mm})$

$r_{1}$ : radius of the cone base $(\mathrm{mm})$

$r_{2}$ : radius of the immersed position of the cone $(\mathrm{mm})$

$R$ : radius of the circular tank (mm)

$t$ : time $(\mathrm{h})$

$T:$ torque $(\mathrm{Nm})$

$V$ : total volume of water $(\mathrm{L})$

\section{Greek Symbols}

$\theta$ : half tip angle of the cone $\left({ }^{\circ}\right)$

$\omega$ : rotation rate of the cone (rpm)

\section{Subscript}

i : initial value

s : saturation value 


\section{Introduction}

In closed water systems such as dams, lakes and marshes, the water in the surface layer is warmed by thermal radiation from the sun and stays in the top layer. Therefore, a warm surface layer is established on the top, while a relatively cold layer is inevitably established at the bottom. Consequently, the warm water remains in the surface layer and the cold water in the bottom one, since a thermal stratification is formed in the vertical direction. In such a situation, organic nutritive elements like phosphorus and nitrogen are increased in the surface layer and eutrophication is enhanced, which leads to occurring of algal blooms in the water. Eventually, the water quality gets worse due to a bad smell, muddiness, and so on. An efficient solution to make the water quality better is to improve purification ability of nature by activating an ecological system inside the closed water area. This implies that a concentration of dissolved oxygen must be increased by promoting a mass transfer of oxygen from the air to the water.

Aeration plays an important role in enhancement of the oxygen mass transfer. Many different types of aeration systems have been developed over the years. It is common in the aeration to pass the air through the water with the Venturi tube, aeration turbines, compressed air, etc. In order to evaluate the performance of different types of aeration systems, a standard for the measurement of the oxygen transfer in clean or tap water is established by American Society of Civil Engineers(ASCE) based on the basic model of Brown et al. (1982), where the standard describes in detail the experimental apparatus and methods, and recommends the standard conditions such as zero dissolved oxygen level, $20{ }^{\circ} \mathrm{C}$ water temperature and 1 atm pressureASCE (2006).

On the studies using air bubbles for aeration, Ashley et al. (1992) have studied benchscale study of oxygen transfer in coarse bubbles, while Duchene et al. (2001) have focused on fine bubbles for the aeration. A range of diameter of coarse bubbles is between 6-10 $\mathrm{mm}$ and one of fine bubbles is between $2-5 \mathrm{~mm}$. They showed that these bubbles were easy to create without much power and energy, and effective for the aeration. Chen et al. (2003) have investigated an aeration process to obtain high oxygen dissolution applying a gas-inducing reactor, where pure oxygen was introduced into the reactor and was stirred with 6-blade pitched-blade downward turbine. They obtained a correlation 
regarding agitation power consumption and oxygen mass transfer coefficient. In addition, Yamada et al. (2005) have proposed a method to improve the water quality using microbubbles whose sizes are micro order. They studied the relation between distributions of micro-bubbles and dissolved oxygen concentrations, and showed that the method using micro-bubbles was by far advantageous to supply oxygen into the water. It should be noted, however, that a large quantity of energy must be required to create the microbubbles. Furthermore, Chen and Yu (1997) have tried to estimate an emission rate of volatile organic compounds instead of the water from the aeration systems. Chern et al. (2001) and Rosso and Stenstrom (2006) have studied effects of impurities and surfactants on the oxygen transfer rate.

As mentioned above, the aeration systems which directly pass the air into the water have been extensively investigated. Contrary to the aeration systems using air bubbles, several attempts have been made for liquid surface aeration. Srinivasan and Aiken (1988) have investigated the mass transfer and physical absorption in liquids dispersed as droplets in a gas. The droplets were formed by the break up of a cylindrical jet with a complex and turbulent mechanism. They claimed that the convection due to the formation process significantly enhanced mass transfer. McWhirter et al. (1995) have developed a fundamentally rigorous oxygen mass transfer model using surface aerator with aerator blades. The model separated the oxygen transfer process into a liquid spray mass transfer zone and a surface reaeration mass transfer zone, which was able to provide the methodology and techniques for quantitatively determining the oxygen transfer rate within each of these important but fundamentally different oxygen mass transfer zones. Chern and Yang (2004) have studied oxygen transfer rate in a tank with water in a drop structure, where the water droplets were generated from a nozzle and a recirculation was established because the water was supplied by pumping up into the nozzle. They examined semiempirical equations to correlate the parameters of the suggested oxygen model as functions of water recirculation rate, drop height, water depth and temperature.

In this study, we use a mist flow composed of smaller water droplets for the aeration. It is common to use a liquid jet from a nozzle driven by a high pressure generated with devices such as fans, compressors, pumps and so on. However, the system based on the liquid jet becomes large and inefficient because these several devices are needed. In addition, it is 
difficult to control the characteristics of the atomization, i.e. droplet diameter, quantity of the mist flow, etc. Therefore, a new atomization system for generating droplets is required, which should be compact, electricity saving and easily controllable. We propose a new atomization system which uses an interesting flow phenomena that the liquid comes rising along the outer(not the inner) surface of a rotating cone, where the cone is immersed in the liquid by turning the top upside down. The liquid rising along the outer surface becomes thinner and forms a film flow, leading to atomization of the liquid. We call the interesting flow phenomena a pumping-up mechanism caused by the rising film flow. Indeed, it is comprehensible and well known that the liquid rises along the inner surface of a rotating hollow cone due to the centrifugal force( Bruin et al. (1969), Makarytchev et al. (1997) and Makarytchev et al. (2001)), but there is only a research of Adachi et al., $2010(\mathrm{a}, \mathrm{b})$ on the phenomena that the liquid rises along the outer surface of the rotating cone and does not separate from the surface. Recently, Adachi (2013) and Adachi and Arai (2011) have examined the thin film pumping-up mechanism on oxygen mass transfer. They showed that the mist flow generated by the mechanism was effective for an aeration.

In this paper, we experimentally investigate the oxygen mass transfer and required power consumption with the new atomization system which uses the pumping-up mechanism caused by the rising film flow along the outer surface of the rotating cone. First, we show the results of the visualization of the interesting flow phenomena along the cone with a high-speed video camera. Then, the dissolved oxygen concentrations are measured for varying rotation rates as $500 \leq \omega \leq 2500$ and the oxygen transfer rates are calculated using the measured date. In addition, the torque acting on the axis of rotation is measured to obtain a correlation between the unit volume power consumption and the oxygen mass transfer.

\section{Experimental set up}

Figure 1(a) shows the experimental apparatus. In the test section, a cone is set at the center of a circular tank with an inner radius of $R=340 \mathrm{~mm}, h_{2}=900 \mathrm{~mm}$ and filled with municipal tap water of a height of $h_{1}=130 \mathrm{~mm}$ (about $50 \mathrm{~L}$ ) as a working fluid. Dimensions of the cone are shown in Fig. 1 (b), where a half tip angle is $=30^{\circ}$ and a maximum radius at the bottom of the cone is $r_{1}=30 \mathrm{~mm}$. The cone is connected to a 
disk with a diameter of $r_{0}=150 \mathrm{~mm}$ and $1 \mathrm{~mm}$ thickness. The disk is useful for making the liquid droplets much smaller. It should be noted that the quality of the material of the cone is an Acrylonitrile Butadiene Styrene (ABS) copolymer and a special treatment is not given to the surface of the cone. The cone is immersed in the water such that the immersed radius is $r_{2}=10 \mathrm{~mm}$. When the cone rotates, a film flow of the water rises along the outer surface of the cone and reaches the base of the cone. After that, the film flow goes along the upper disk connected to the cone and radiates outward being atomized.

The experimental procedure for all tests follows the ASCE Standard for Measurement of Oxygen TransferASCE (2006). At the beginning of each test run, the sodium sulfite of $10 \mathrm{~g}$ is added as a deoxidizer to the tap water of $50 \mathrm{~L}$ and the cone is rotated with a lower rotation rate until the dissolved oxygen reaches zero level. It should be noted that the mixing with the lower rotation rate is continued until the effect of the deoxidizer is vanished. After that, the rotation rate is set to a designated value with the PI(Proportional-Integral) Control. Then, the concentration of dissolved oxygen in the test section is measured by using a DO meter (OM-51, Horiba Inc. Japan) whose probe is put under the water beside the side wall as seen in Fig. 1(a). The sampling rate of the DO meter is set up $1 / 60 \mathrm{~Hz}$. The operation temperature of the water inside the tank is maintained to be $20 \pm 0.5{ }^{\circ} \mathrm{C}$ with a thermal controller and a heater with the PID(Proportional-Integral-Derivative) Control, because a saturation concentration of the dissolved oxygen changes depending on the water temperature. On the other hand, axial toque is measured by using torque sensor(UTM-0.3Nm, Unipulse, Japan), where the sampling rate is $1 \mathrm{kHz}$ and the measurement time is for $10 \mathrm{sec}$.

\section{Results}

\subsection{Visualization of Rising Film Flow and Circulation}

Thin liquid film flow rising along the outer surface of the cone is generated when the immersed cone rotates in the circular container. We visualize the phenomena with a highspeed video camera. Flow patterns of the rising film flow are shown in Fig. 2, where a rotation rate of the cone is gradually changed from $=0$ to $6000 \mathrm{rpm}$. Figure 2(a) shows an initial state. The cone is immersed in the liquid by turning the top upside down and the cone is in a state of rest, where the water surface is indicated by the thick bright 
horizontal line. Once the cone begins to rotate under the relatively small rotation rate such as $500 \mathrm{rpm}$, water is deformed and lifted up at the vicinity of the cone surface as seen in Fig. 2(b). However, the water does not go up anymore at that time because the rotation rate is small. When the rotation rate is further increased, the deformation of the water becomes larger as seen in Fig. 2(c), and the water winding around the cone rises higher due to the larger degree of the deformation caused by the lifting up. After that, a mass of water is scattered out radially in radius and tangential directions. The pumping-up phenomena for the liquid film is generated when the rotation rate exceeds about $1000 \mathrm{rpm}$. Subsequently, the thin liquid film flow is generated which rises along the outer surface on the cone as seen in Fig. 2(d), where the rotation rate is about 6000 rpm. It should be noted that the film flow cannot keep the filmwise condition when the film flow goes up further because the radius of the cone becomes larger and the thickness of the film flow becomes thinner, although it does not separate from the cone surface. Eventually the thin liquid film flow is atomized into a mist flow as seen in the schematic figure of $2(\mathrm{e})$. We have examined the wettability on the phenomena in the preliminary experiment by applying the hydrophilic and hydrophobic solvents on the cone surface. Then, we did not find any fundamental difference except that the different patterns can be seen when the liquid is scattered at the quite early stage of the rising film flow. Namely, the mass of liquid is scattered for the hydrophobic solvent, while it is not scattered for the hydrophilic one. In this study, the cone is connected to the circular disk as seen in Fig. 1(b) and the film flow goes along the disk being thinner, which is useful for making the liquid droplets much smaller. The atomized mist flow from the edge of the circular disk is shown in Fig. 3.

In addition, flow visualization under the cone inside the water is carried out by using the aluminum dust method. A visualization photograph is shown in Fig. 4(a) together with the schematic figure (b) which explains the flow pattern generated by the rotating cone. A light sheet illuminates the vertical plane including the rotating axis. So, we can capture the reflected light from the aluminum dust and recognize the vortex structure. It should be noted that there are no air bubbles in the visualization photograph. We can see a strong vortex filament along the central axis of the rotating cone, where an upward flow is generated due to a spin-up of the rotation of the cone. A part of the upward flow goes 
up along the cone outer surface to generate the rising film flow(A-E in Fig. 4(b)). The other part of the upward flow goes along the water surface outward the wall of the circular tank (A-B), goes down into the bottom along the side wall(B-C) and converges to the center along the bottom wall(C-D). After that, the flow is going up again generating the vortex filament along the central axis(D-A). It should be noted that the so-called Ekman layer is formed on the water surface and the bottom wall, while the Stewartson layer on the side wall. Therefore, there is a large toroidal and helical circulation like a torus inside the water under the cone, which is effective for transport processes.

\subsection{Oxygen Mass Transfer}

In order to evaluate the mass transfer of oxygen from the air to the water through the water droplets in the test section, we define a mass transfer rate. The temporal evolution of a dissolved oxygen concentration $D o \mathrm{mg} / \mathrm{L}$ can be expressed as

$$
\frac{d D o}{d t}=k_{L} a\left(D o_{s}-D o\right)
$$

where $k_{L} a \mathrm{~h}^{-1}$ and $D o_{s}$ are a oxygen mass transfer coefficient and a saturation value of the dissolved oxygen concentration(Brown et al. (1982) and ASCE (2006)). Solving Eq. (1), we obtain the following solution as

$$
D o=D o_{s}-\left(D o_{i}-D o_{s}\right) \exp \left(-k_{L} a t\right)
$$

where $D o_{i}$ is an initial value of the dissolved oxygen concentration. Introducing a shift of time $t^{\prime}$, we can rewrite Eq. (2) as

$$
D o=D o_{s}\left[1-\exp \left\{-k_{L} a\left(t-t^{\prime}\right)\right\}\right]
$$

where the dissolved oxygen concentration is zero as $D o=0$ at $t=t^{\prime}$. The time $t^{\prime}$ is defined as

$$
t^{\prime}=\frac{1}{k_{L} a} \ln \left(\frac{D o_{s}-D o_{i}}{D o_{s}}\right) .
$$

By assuming the form of Eq. (2), the least-square fitting is carried out using NewtonRaphson method and the coefficients of $k_{L} a, D o_{i}$ and $D o_{s}$ are calculated. Then, $t^{\prime}$ can be calculated using the values of the coefficients from Eq. (4). Finally, we can obtain the fitting curve of $D o$ for all experimental results where the initial value is zero, i.e. $D o_{i}=0$ at $t=0$, by shifting the time difference of $t^{\prime}$. 
Under the conditions in our experiments, the mist flow is generated at $1200 \mathrm{rpm}$ when the rotation rate is gradually increased, while the mist flow can be maintained at the lower rotation rate as large as $800 \mathrm{rpm}$ if the rotation rate is decreased gradually from the situation that the mist flow is already generated at the large rotation rate. Namely, there is a hysteresis for the mist generation depending on the manner of the control of rotation rate. This is mainly due to a condition of wettability on the cone surface. If the cone surface is dried, the larger rotation rate is necessary to generate the liquid film because the surface tension between the cone surface and working fluid acts to cause a blocking of film generation. On the other hand, if the cone surface is already wetted, the blocking by the surface tension does not work. Therefore, it is thought that the film flow can be kept even for the smaller rotation rate in the wetted case.

As mentioned above, for example, there are two possible states depending on the manner of the control in the case of $1000 \mathrm{rpm}$. One is the state with the mist flow, and the other is that only the circulation exists without the mist flow. Figure 5 is the results of the time evolution of the dissolved oxygen for $1000 \mathrm{rpm}$. The red solid line shows the time evolution of the dissolved oxygen under the condition with the mist flow, while the blue dashed line under the condition without the mist flow, where the dissolved oxygen is normalized as $D o / D o_{s}$ using its saturation value. In the case without the mist flow, the oxygen transfer is surface aeration and transported only due to the circulation formed inside the water under the rotating cone. On the other hand, once the mist flow is generated, the oxygen transfer is enhanced, by a double advantage, due to the circulation and mist flow. As seen in Fig. 5, the elapsed time which is needed to reach its saturation point from zero concentration of the dissolved oxygen is about 25 hours in case without the mist flow, while the time is shorter and about 10 hours in case with the mist flow.

The oxygen mass transfer is affected by the values of rotation rate because the flow rate of the mist flow and the intensity of the circulation under the cone change depending on the rotation rate. So, we measure the temporal evolution of the dissolved oxygen concentration for varying rotation rates, calculate the oxygen mass transfer coefficient $k_{L} a$ from the measured data and depict the values in Fig. 6 against the rotation rates. We can see that the values of the coefficient is smaller for the case without the mist flow and the smaller values continue up to $1200 \mathrm{rpm}$ when the rotation rate is increased from 
the sufficiently small value. The mist flow is generated when the rotation rate exceeds $1200 \mathrm{rpm}$, which leads to the increase of the oxygen mass transfer coefficient because the oxygen transfer is enhanced due to the dual effect of the circulation and mist flow. It is evident that the mist flow apparently enhances the oxygen transfer from the oxygen transfer coefficients. If the rotation rate is decreased gradually from the situation that the mist flow is already generated, the mist flow is terminated at $800 \mathrm{rpm}$ and the coefficient is decreased abruptly there. Therefore, the hysteresis phenomena as mentioned previously can be also seen here. It should be compared the oxygen mass transfer rate to the previous our results in Adachi and Arai (2011). In our previous study, the tank size was 11[L] which was smaller than the present tank, and the oxygen transfer rate was $2 \sim 9\left[\mathrm{~h}^{-1}\right]$ for the range of rotation rate as $1000 \sim 1500$ [rpm]. It should be noted that the oxygen mass transfer rate becomes larger if the size of the test section is smaller.

On the other hand, we can see that the coefficient becomes slightly larger when the rotation rate exceeds $1500 \mathrm{rpm}$, where a slope of $k_{L} a$ increases against the rotation rate. This is mainly because the flow pattern inside the test section is changed. Namely, the flow inside the circular tank is a steady state flow and like a free vortex with a strong vortex filament along the central rotational axis for the smaller rotation rates as seen before in Fig. 7, while the flow pattern is undergone to an unsteady flow and whole water inside the tank comes to move as a rigid-body rotation like a forced vortex for larger rotation rate than $1500 \mathrm{rpm}$. In such situation of the forced vortex, waves with quite large amplitudes appear on the water surface as shown in Fig. 7. Figure 7 shows the unsteady flow patterns for a half cycle at every one-fourth cycle from the arbitrary time for the rotation as large as $1500 \mathrm{rpm}$. In Fig. 7(a), we can see that the larger amplitude of the surface wave is formed on the left side. As one-fourth cycle passes, the larger amplitude part moves on the front face. After that, as one-fourth cycle further passes, the larger amplitude part moves to the right side. From the literature of Alekseenko et al. (1994), an essential effect of the waves is seen at the heat and mass transfer through the free surface because the gas is entrained. Therefore, in our case, unsteady wavy flow has also a possibility to exert not a little influence to oxygen mass transfer augmentation. Namely, it is thought to be that such waves with larger amplitudes might roll up the oxygen and slightly enhance the oxygen mass transfer for the unsteady flow regime. 
For the practical use of an aeration system, it is important to know the required power consumption. So, we measure axial torque acting on the rotating cone for varying rotation rates. First, we measure a reference torque, where the cone is not immersed in the water. Subtracting the reference torque from the axial torque acting on the rotating cone which is immersed in the water, we obtain the net torque requirement to mix and atomize the liquid by the rotating cone. Figure 8 shows the net torque $T$ as a function of the rotation rate $\omega$. It is evident that the torque is small when the mist flow is not generated, which means that only the small torque is needed to make the circulation inside the water. On the other hand, once the mist flow is generated, the torque becomes larger because the pumping-up and atomization of the liquid as well as the circulation inside the water appear along the rotating cone and rotating circular plate.

Finally, we show a correlation between a unit volume power consumption $P / V$ and the oxygen mass transfer $k_{L} a$ in Fig. 9 together with the result of Chen et al. (2003), where $P$ is a net power consumption and $V(50 \mathrm{~L})$ is a volume of the working fluid. In the case without the mist flow, the power consumption per unit volume is small, and the oxygen mass transfer is also small. In the case with the mist flow, the oxygen mass transfer seems to be linearly proportional to the unit volume power consumption in the logarithmic graph. Actually, the correlation between the unit volume power consumption and the oxygen mass transfer is examined. It is found that the slope in the logarithmic graph is 0.328 , and the oxygen transfer increases in power law relationship with the unit volume power consumption such as $k_{L} a \sim(P / V)^{0.328}$. Chen et al. (2003) reported a correlation of $k_{L} a$ as a function of $P / V$. It might not be appropriate to compare our results with their ones, because they used pure oxygen bubbles for aeration and stirred the water system with 6-blade pitched-blade downward turbine. Their system needed much power consumption although large oxygen mass transfer coefficient was obtained. The important thing is that their result of the oxygen mass transfer coefficient must be quite larger value than our results since they used pure oxygen. Nevertheless, it is noteworthy that if the necessary power consumption is as small as $P / V \sim 10^{-2}$, the oxygen mass transfer coefficient is possibly comparable with our results in the case with the mist flow. It is expected that the efficiency of our system is larger for the range of $P / V<10^{-2}$. 
1 This suggests that our system is efficient for the case that the power consumption is small 2 and/or the volume size is large. The power consumption necessary for spraying the mist 3 flow might be constant independent of the volume size because the cone is rotating just 4 on the water surface, while the oxygen can be well transported due to the vortex filament 5 even if the volume size is large. In the range of the mist flow, we perform the least square 6 fit and obtain the power law relationship between the oxygen transfer and the unit volume 7 power consumption such as $k_{L} a \sim 3.14(P / V)^{0.328}$.

8

\section{Conclusions}

We have experimentally investigated oxygen mass transfer and power consumption for a new aeration system by using a rotating cone. The interesting phenomena of pumpingup mechanism, rising liquid film flow, circulation of vortex filament and atomization have been shown in the visualization photographs. The series of atomization mechanism has been applied to the new aeration system. It should be emphasized that the method is very simple and easier technique to make the mist flow than the existing ones. In addition, we have measured the mass transfer coefficient of the oxygen transfer from the air to the water with the new atomization device and necessary power consumption for the variations of the rotation rates. We have shown the potential development of the new atomization device using the rotating cone in purification of water quality. We summarize the main conclusions as follows.

1) It is shown that a large circulation with the vortex filament is generated inside the water by rotating a cone, where the circulation forms a helical flow like a torus inside the water under the cone. On the other hand, a thin liquid film flow is generated, which rises along the outer surface on the rotating cone. The film flow is eventually atomized into a mist flow.

2) It is found that the oxygen mass transfer coefficient which is calculated from the measured data of dissolved oxygen concentration is larger for the case with the mist flow than for the case without the mist flow. It is shown that the mist flow is effective for the oxygen mass transfer by adding it to a circulation.

3) The flow pattern has been changed from the steady state free vortex to the unsteady forced vortex if the rotation rate is grater than about 1500. It is found that the oxygen 
1 mass transfer is enhanced in the unsteady flow regime, because surface waves with quite 2 large amplitude is generated and promotes air entrainment on the water surface.

3

4) It is found that the correlation between the unit volume power consumption and 4 the oxygen mass transfer is examined and shows that the oxygen transfer increases 5 in power law relationship with the power consumption in the case with the mist flow 6 such as $k_{L} a \sim 3.14(P / V)^{0.328}$.

As previously mentioned, the motivation of the present work is to study the water 8 purification in closed water systems like dams and lakes. In such water systems, the 9 depth of water is high, so a thermal stratification is formed in the vertical direction, which causes the water pollution. Using our aeration system, water droplets absorbing 1 oxygen can be transported into the deep area in the water system because vortex filament, Ekman layer and Stewartson layer are generated and promote the oxygen transfer. So, we would like to highlight that out system is expected to have an advantage of efficient aeration system if applied to the deep water system.

\section{Acknowledgments}

The author expresses his cordial thanks to Mr. K. Matsuda and Mr. T. Kubo for 17 valuable discussion. The author expresses his cordial thanks also to Prof. M. Terashima and Technology, (2) A-STEP Program 10101745 from Japan Science and Technology

1 Agency and (3) the ISM Cooperative Research Program (2013-ISM-CRP-2088). 


\section{References}

Adachi, T., Sato, N., Kobari, N. and Hori, T., 2010(a), Liquid Film Flow Rising along the Outer Surface of the Rotating Cone The Japan Society of Mechanical Engineering Series B (in Japanese), 76-761, 161-163.

Adachi, T., Sato, N., Kobari, N. and Hori, T., 2010(b), Liquid Film Flow Rising along the Outer Surface of the Rotating Cone, Heat Transfer-Asian Research, 39, 492-496.

Adachi, T., and Arai, A., 2011, Mist Type Purifier Using Pumping-up Mechanism Generated by Thin Film Flow, Proceedings of 24th European Conference on Liquid Atomization And Spray Systems, CD-ROM.

Adachi, T., 2013, Enhancement of Oxygen Dissolution Using Mist Flow Generated by a Rotating Cone, The Japan Society of Mechanical Engineering Series B (in Japanese), 79-800, 632-635.

Alekseenko, S. V., Nakoryakov, V. E., and Pokusaev, B. G., 1994, Wave flow of liquid films Begell House, Inc., $§ 13$.

ASCE, Measurement of Oxygen Transfer in Clean water, America Society of Civil Engineers, 2006.

Ashley, K., I., Mavinic, D., S., and Hall, K., J., 1992, Bench-scale Study of Oxygen Transfer in Coarse Bubble Diffused Aeration, Water Research, 26, 1289-1295.

Brown, L. C., and Baillod, C. R., 1982, Modeling and Interpreting Oxygen Transfer Data, ASCE, J. Envir. Engrg. Div., 108(EE4), 607-628.

Bruin, S., 1969, Velocity Distributions in a Liquid Film Flowing over a Rotating Conical Surface, Chemical Engineering Science, 24, 1647-1654.

Chen, J-H., and Yu, C. F., 1997, Oxygen Transfer Modeling of Diffused Aeration Systems, Ind. Eng. Chem. Res., 36, 5447-5453.

Chen, J-H., Hsu, Y-C., Chen, Y-F., and Lin, C-C., 2003, Application of gas-inducing reactor to obtain high oxygen dissolution in aeration process, Water Research, 37, 29192928. 
2 Chern, J-M., Chou, S-R., and Shang, C-S., 2001, Effects of Impurities on Oxygen Transfer Rates in Diffused Aeration Systems, Water Research, 35, 3041-3048.

4 Chern, J-M., and Yang, S-P., 2004, Measuring and modeling of oxygen transfer rate in a drop structure, Ind. Eng. Chem. Res., 43, 7657-7663.

6 Duchene, P., Cotteux, E., and Capela, S., 2001, Applying fine bubble aeration to small tanks, Water Science and Technology : a Journal of the International Association on Water Pollution Research., 44, 203-210.

9 Makarytchev, S. V., Langrish, T. A. G. and Prince R. G. H., 1997, On Modeling Fluid over a Rotating Conical Surface, Chemical Engineering Science, 52, 1055-1057.

Makarytchev, S. V., Langrish, T. A. G. and Prince R. G. H., 2001, Thickness and Velocity of Wavy Liquid Films on Rotating Conical Surfaces, Chemical Engineering Science, 56, 77-87.

Mcwhirter, J. R., Chern, J-M., and Hutter, J. C., 1995, Oxygen mass transfer fundamentals of surface aeration, Ind. Eng. Chem. Res., 34, 2644-2654.

Rosso, D., and Stenstrom M. K., 2006, Surfactant effects on $\alpha$-factors in Aeration Systems, Water Research, 40, 1397-1404.

Srinivasan, V., and Aiken, R. C., 1988, Mass transfer to droplets formed by the controlled breakup of a cylindrical jet - physical absorption, Chemical Engineering Science, 43, 3141-3150.

Yamada, S., Amano, T., and Minagawa, H., 2005, A Study for Distribution of Microbubbles and Effects of Oxygen Supplying into Water, The Japan Society of Mechanical Engineering Series B (in Japanese), 71-705, 1301-1306. 


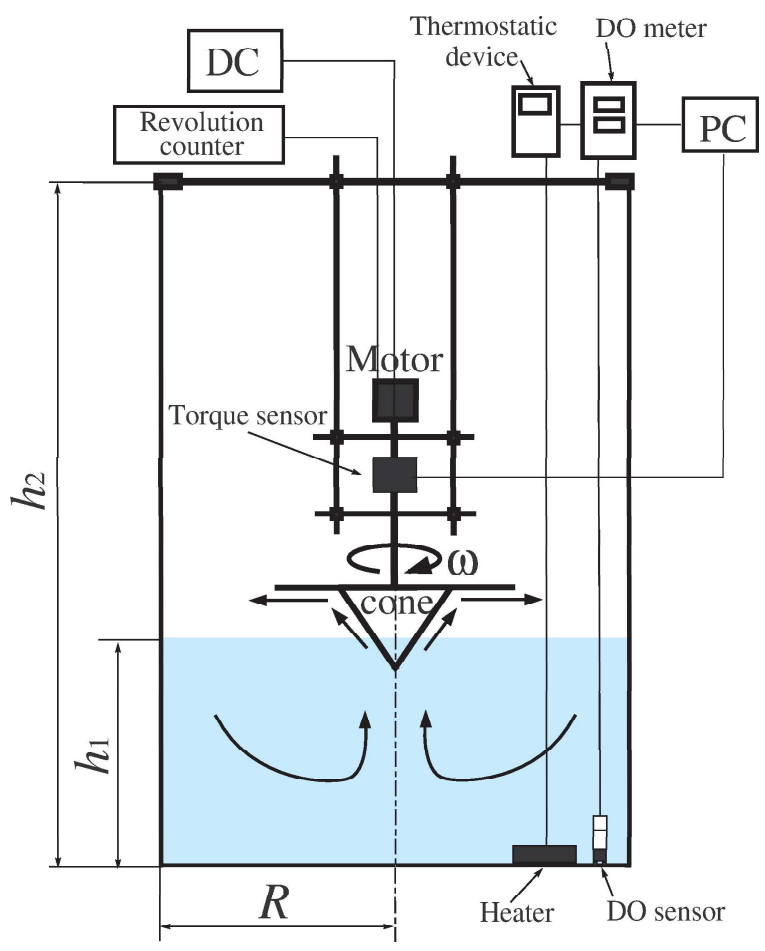

(a)

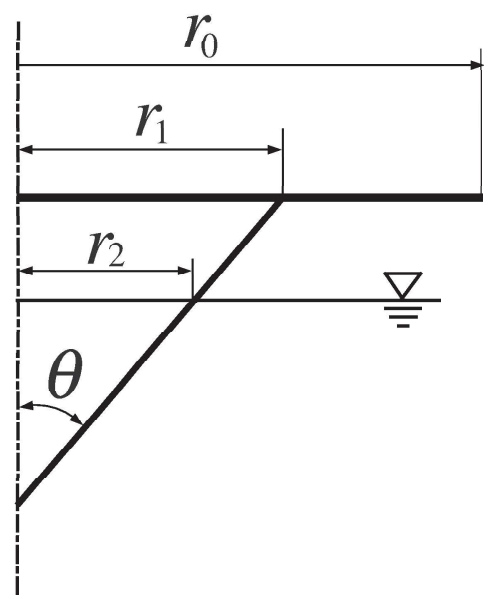

(b)

Figure 1: Experimental apparatus. 


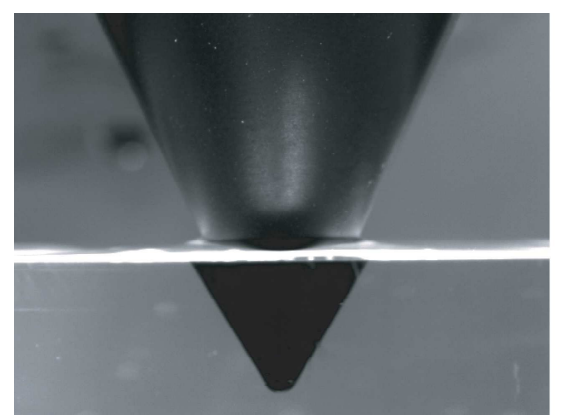

(a)

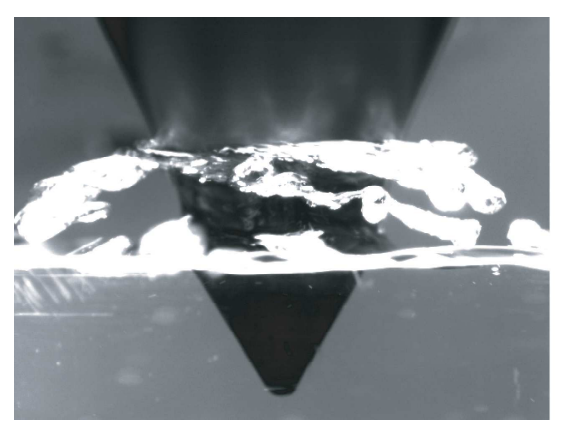

(c)

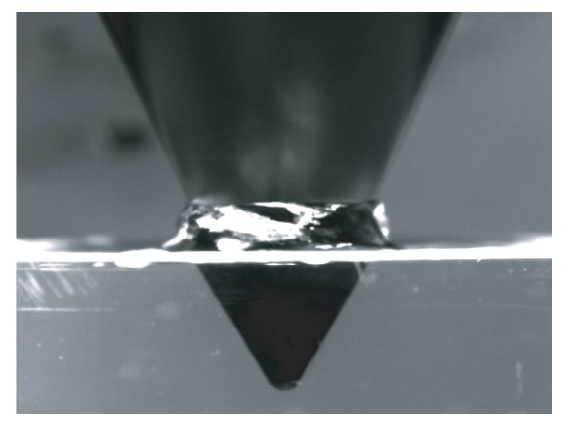

(b)

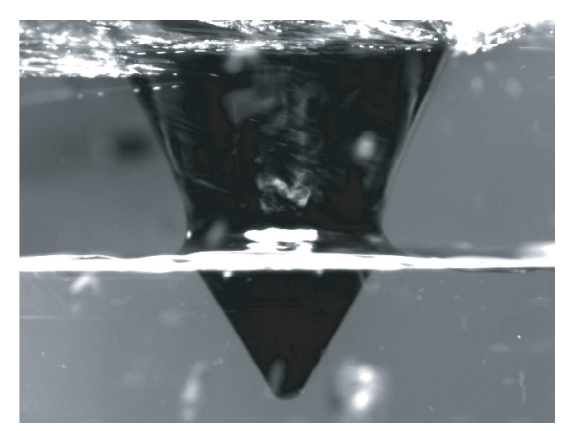

(d)

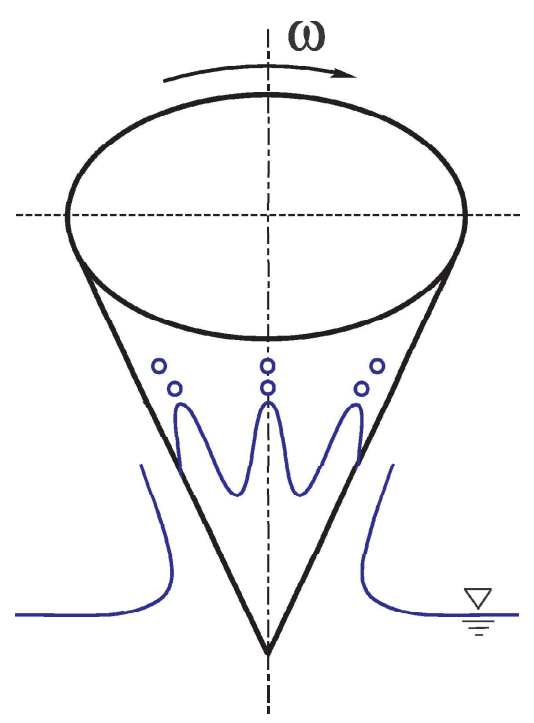

(e)

Figure 2: Visualization photographs of rising film flow. 


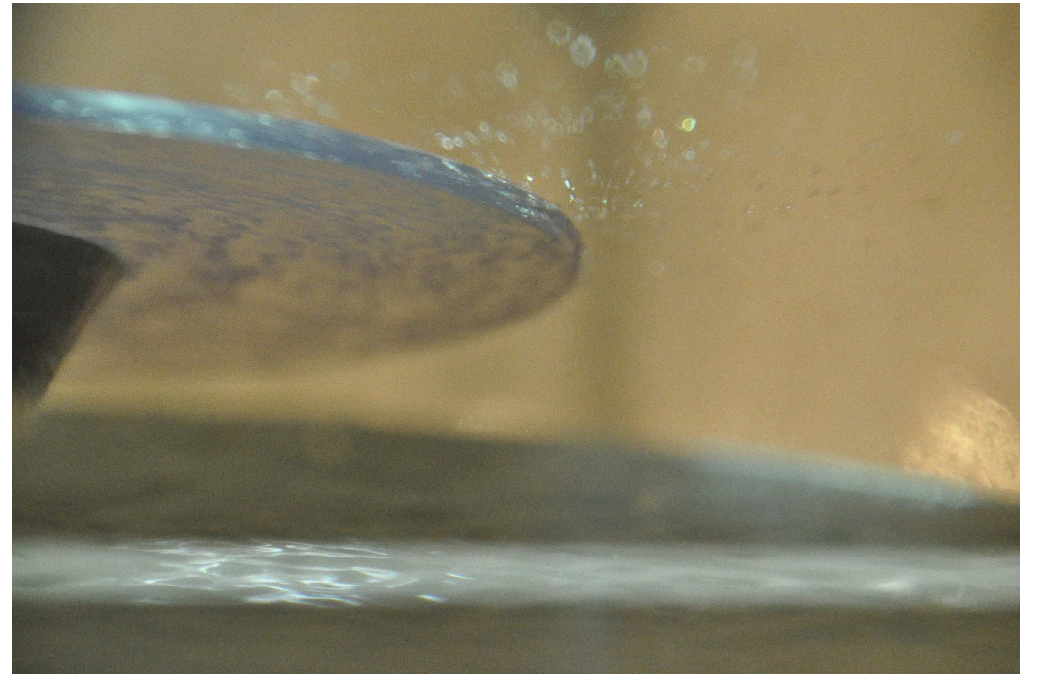

Figure 3: Visualization photograph of mist flow sprayed from the disk edge. 


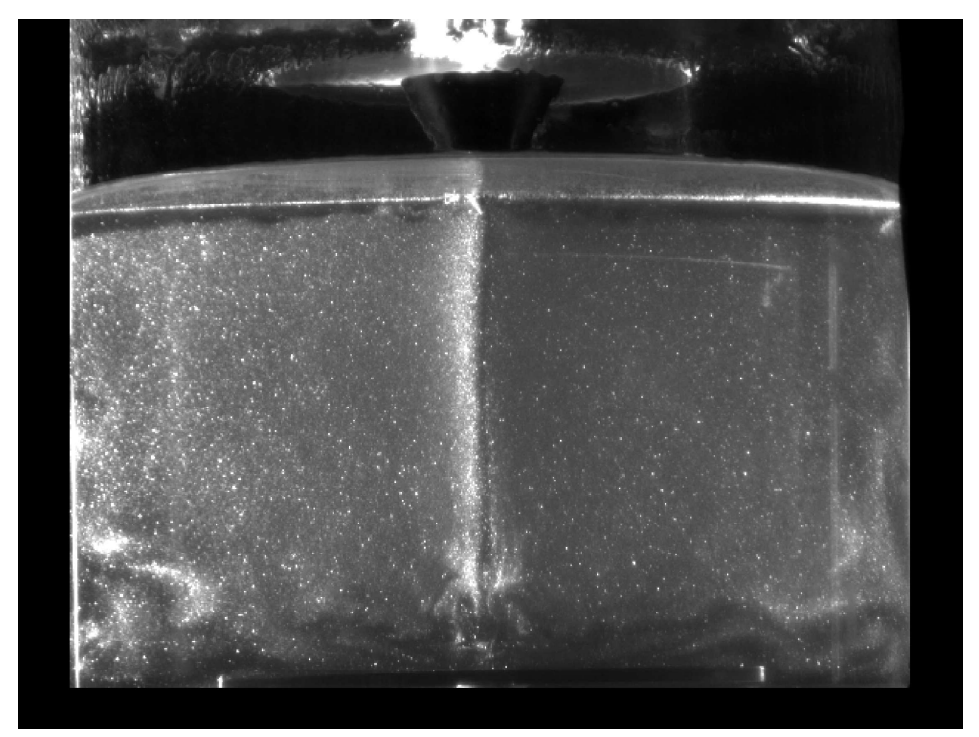

(a)

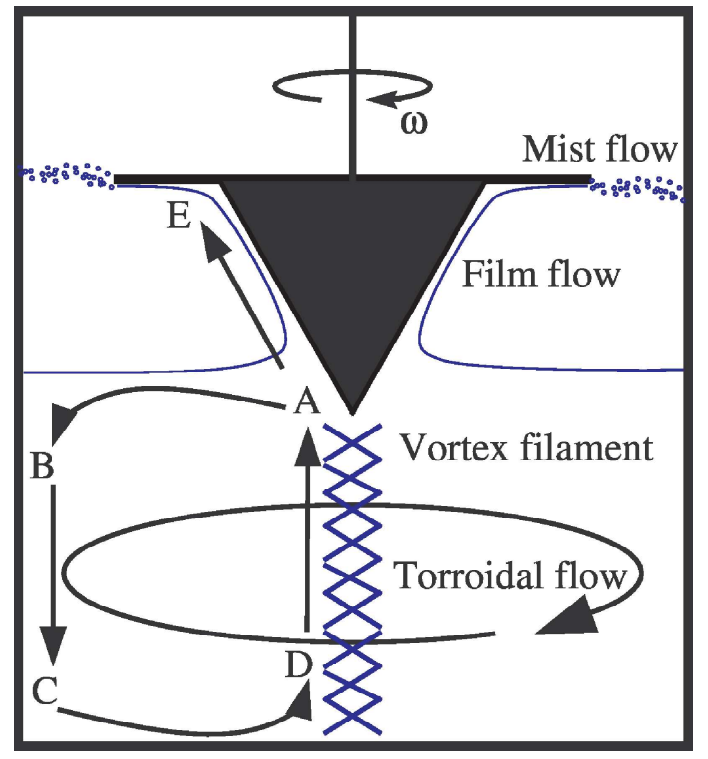

(b)

Figure 4: Visualization photograph of circulation inside the water. 


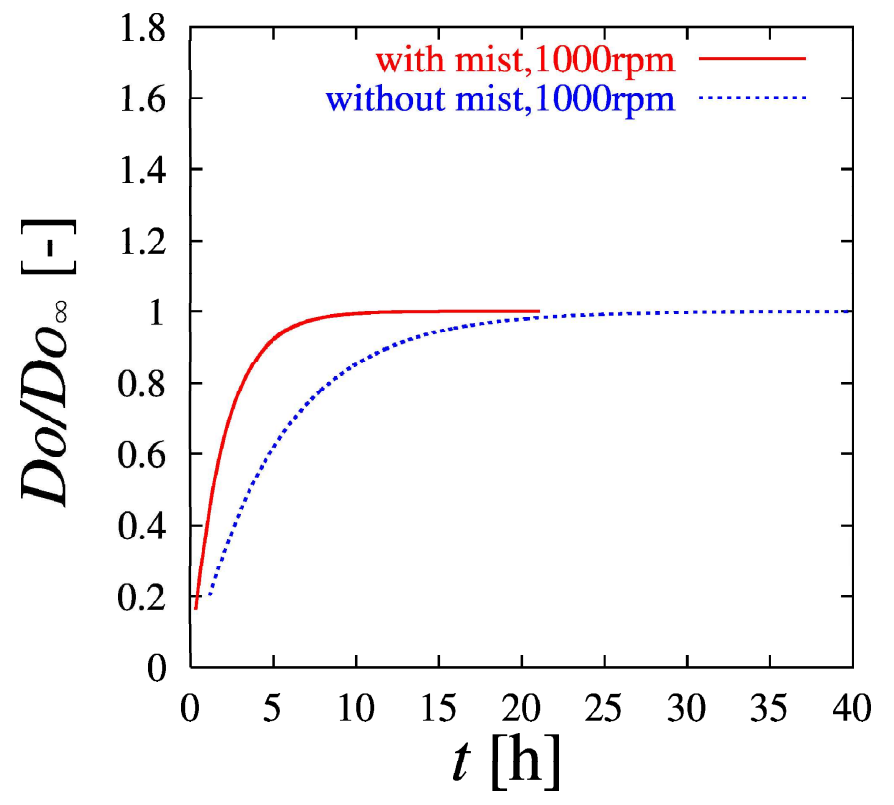

Figure 5: Dissolved oxygen $D o / D o_{s}$ vs. time $t$ 


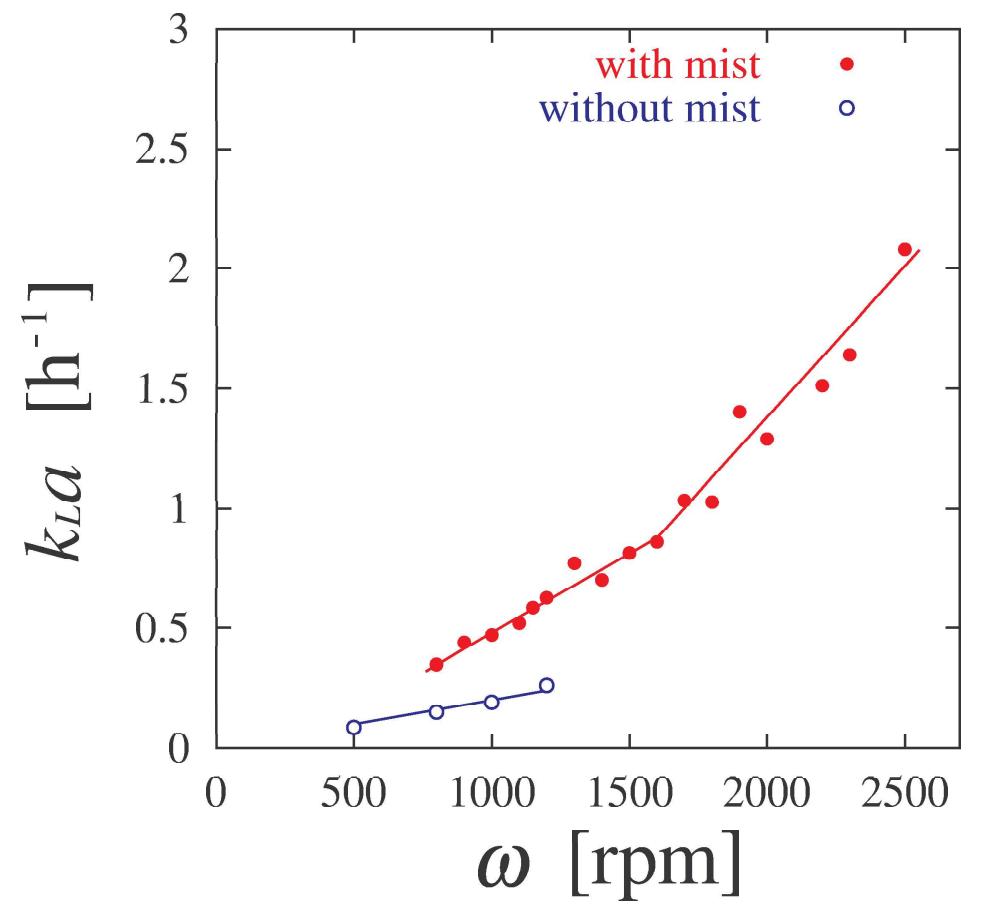

Figure 6: Oxygen mass transfer coefficient $k_{L} a$ vs. rotation rate $\omega$. 


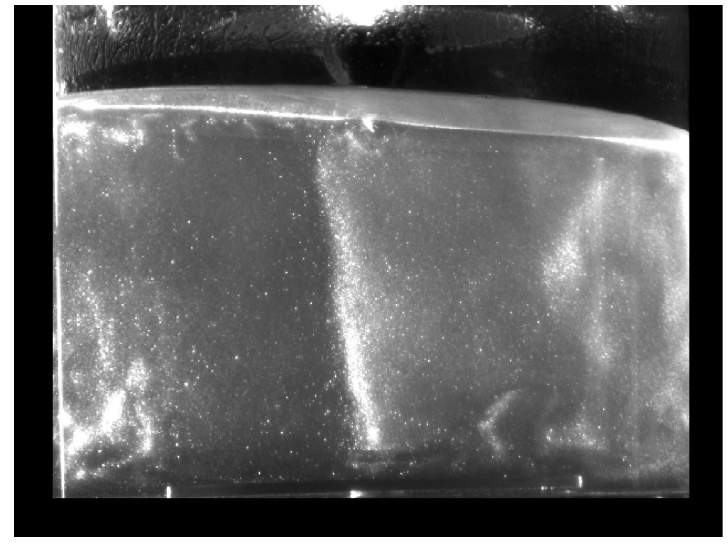

(a)

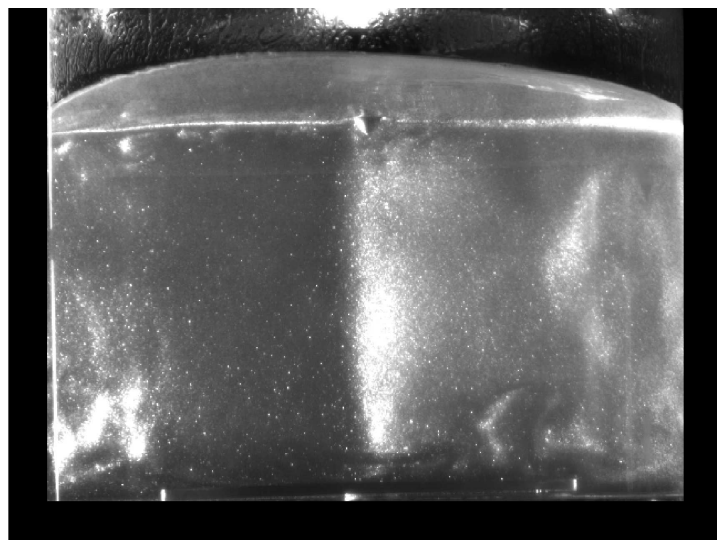

(b)

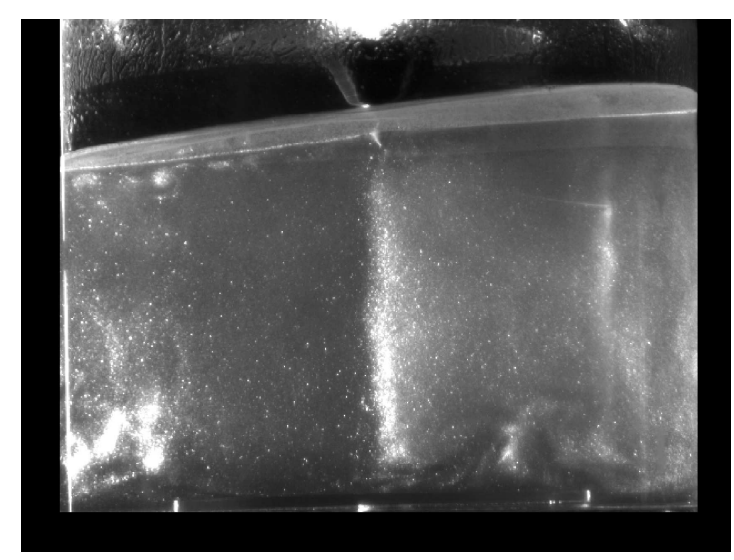

(c)

Figure 7: Visualization photographs of surface wave flow. 


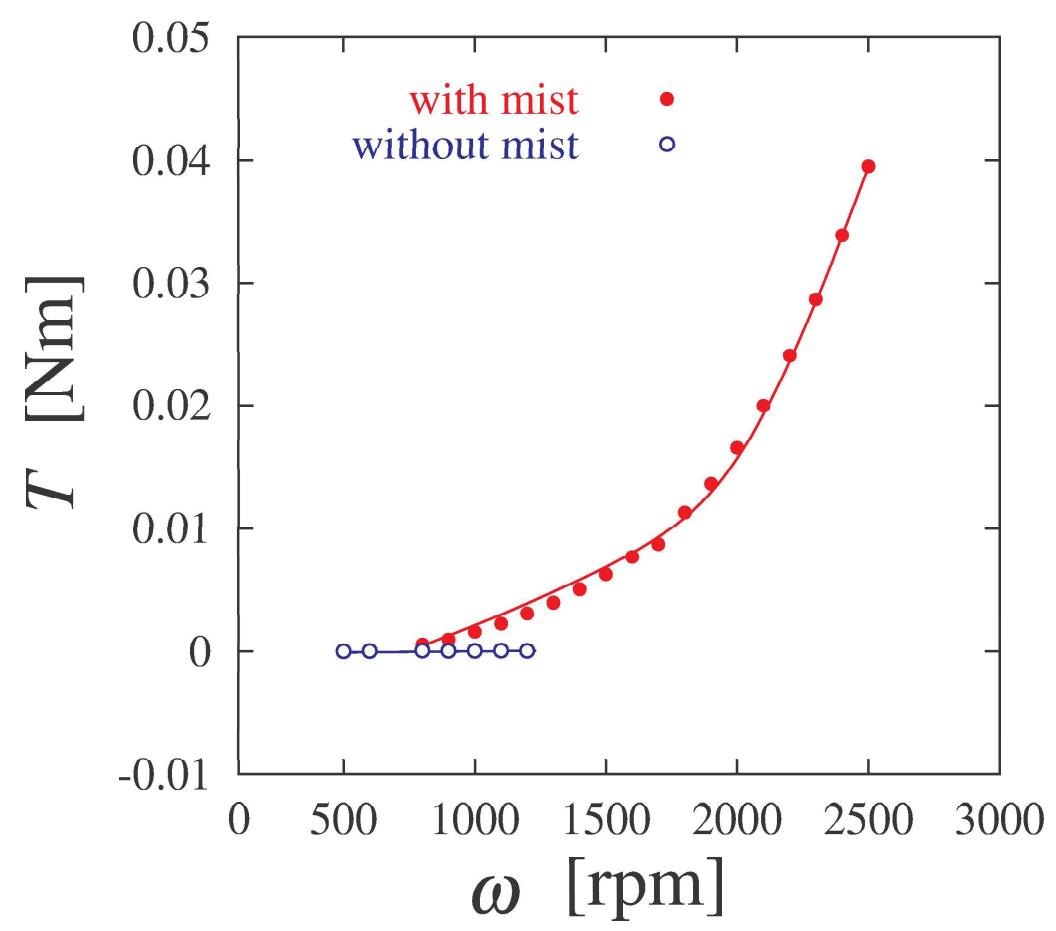

Figure 8: Axial torque $T$ vs. rotation rate $\omega$. 


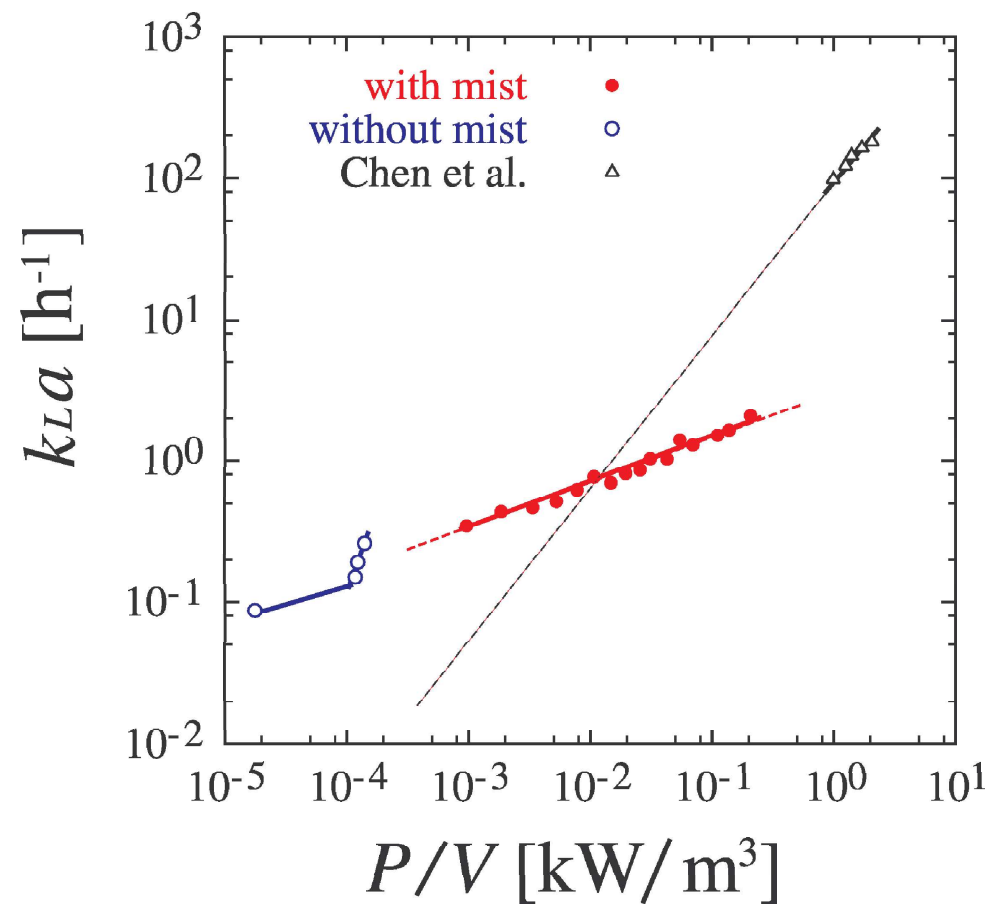

Figure 9: Oxygen mass transfer coefficient $k_{L} a$ vs. unit volume power consumption $P / V$. 
Graphical Abstract

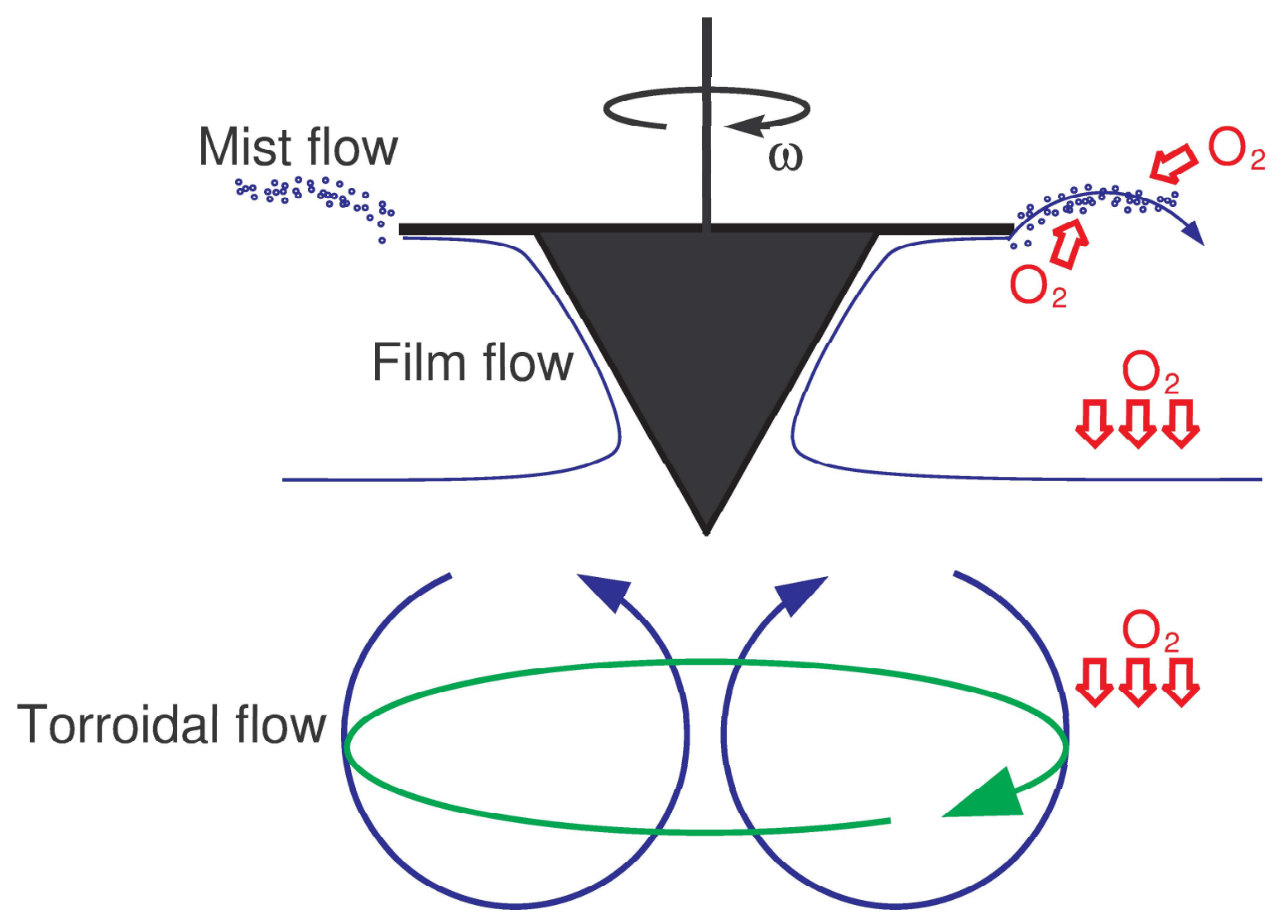

